\title{
Survey of Quality of Service Routing Protocol in MANET
}

\author{
Sarita Soni \\ Research Scholar \\ KNIT Sultanpur \\ INDIA
}

\author{
Samir Srivastava \\ Associate Professor \\ KNIT Sultanpur \\ INDIA
}

\begin{abstract}
In an ad-hoc network's there is no specific infrastructure and no static topology. It has more dynamic topology that changes over time and less battery power of the nodes, less bandwidth and transmission quality enhancements. It supported Real time \& multimedia application by Manet. QOS have parameter like as easy bandwidth utilization, less delay, minimum packet loss, good throughput, jitter. Goal of QoS is to optimized a more positive network conduct, therefore that data carried by the network can be better utilized. and it may minimize of the one way network delay. Delay variance(jitter) and packet loss. Routing is implicit problem in manet because of without of any fixed base station and capricious mobility of nodes rooted onto the best effort distribution of services. In this paper we defines some protocols such as CEDAR,PLBQR,QOLSR, QOS AODV,AND TBP, which is minimize the packet loss, delay, low jitter. A QoS enabled routing protocol is expected to support several matrices with end to end delay, throughput, bandwidth and jitter as well as packet delivery ratio. In QoS some parameter like as easy bandwidth utilization, less delay, minimum packet loss, good throughput etc.
\end{abstract}

\section{Keywords}

mobile ad-hoc network, quality of service, matrices, protocol like CEDAR,OLSR,TBP,AQOR.

\section{INTRODUCTION}

MANETIt is a temporary network, mobile: freely moving network participants (nodes). It is a shared wireless network without any infrastructure consistency of mobile nodes connected by wireless links. The nodes are released to movement and or manage those arbitrarily. NO one premise infrastructure (i.e. backbone routers ).Constantly improving network framework (topology) infusion by multi-hopes between nodes usually wireless networks. End to end delay or single way delay refers of the time taken stand for a packet to be transmitted across a network from source to destination. Manet are used in various and varied application like setting up of conference, e-classroom, patient monitoring, detection of earthquakes etc. Real time and multimedia application supported by Manet, which is also supported in QoS.

We introduce a mode reservation-rooted routing and signaling multiple partition algorithm, Ad hoc QoS on-demand routing (AQOR), that provides end-to-end quality of service (QoS) adherence, in period of bandwidth and end-to-end delay, in mobile ad hoc networks (MANETs). The higher use of MANETs stand for translocation multimedia applications such as voice, video and data, leads to the require to given QoS adherence. It perform correct admission control and resource reservation in AQOR, we have developed elaborate enumeration that permit us of computation the bandwidth and end-to-end delay in unsynchronized wireless existing environment. AQOR also involves accomplished mechanisms stand for QoS maintenance, inclusive temporal protection and target-begin recovery processes. The performance of AQOR is intended into elaboration from pretense using OPNET Modeller. This outcome validate that AQOR gives QoS maintenance in ad hoc wireless networks with high reliability and low overhead. In this we emphasis on giving shortest path routing and high availability into a moveable network atmosphere whither the network topology changes rapidly. QoS support in Manet, the link state information such as delay, bandwidth, shortest path, cost, loss rate, in the network should be manageable and available.

QoS Routing Mechanism:-The main purpose of quality of service is to find a feasible path through the network, and it providing the limited resources to find the QoS requirement. Requirement of qos for real traffic are maximum delay threshold, minimum bandwidth threshold and constant jitter. It is very complex to design and implement routing protocol that can be optimal path in each situation.

- Proactive routing: QOLSR

- Reactive routing: QoS-AODV

- Ticket-based Routing: TBP

- Hierarchical Routing: CEDAR

- Predictive Location-rooted routing: PLQBR

- $\quad$ Power aware routing

Proactive Routing:-In this protocol every node manage single or much tables inclusive routing information to every other node in the network. Or we can say that it maintains the fresh list of destinations and their routes by periodically distributes their routing tables throughout the network. example-Optimized Link State Routing Protocol(OLSR), QOLSR,

Disadvantages

- Respective amount of data for maintenance.

- Slow reaction and restructuring and failures.

Hierarchical State Routing:-In this a multilevel clustering and logical partitioning of mobile nodes.

Reactive Routing:-Routes are created as and when required. In this find a route on demand by flooding with route request packet. QOSAODV

Disadvantages:- High latency time in route finding. 


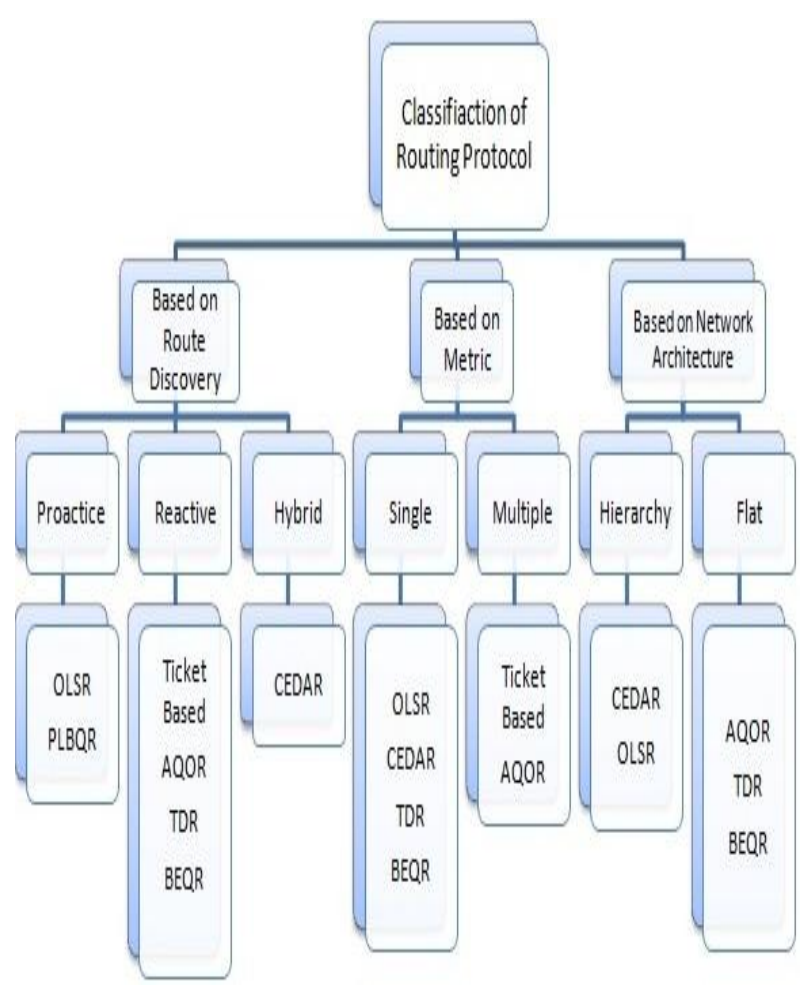

Figure.1 Classification of Routing Protocol

\section{ISSUES AND CHALLENGES FOR QOS PROTOCOL IN MANET}

Unreliable channel:- Wireless connection's high bit rate is might be profound in Manet. It is shared the end to end path through several session. The channel by which the terminal communicate is mainly to noise, Intervention, and Dwindle, and it has short bandwidth compare than a wired network. In some scenario. The path between user's any pairs can traverse multiple wireless connection and the connections themselves can be different. Packet error can be pull-off through forward error correction, retransmission technic. Nevertheless packet error can rise to connection abortiveness, forward to rerouting, lesser throughput, higher packet delay, and packet defection due to overcrowding.

Maintenance of route:- Moveable nature of network topology and changing behavior of infusion moderate makes the maintenance of network state information is very complex. The installed routing path can be split even during the procedure of data transfer. Consequently the need for maintenance and rearrangement of routing paths by minimal overhead and delay factor. The QoS aware routing would search for the reservation of resources at the interlude nodes.

Mobility of the node: After the nodes considered this way are mobile nodes, So that is they movement independently and randomly into all direction speed, the topology knowledge has to be updated repeatedly and accordingly so as to provide to reach the ultimate destination which result in future less packet delivery ratio.

Lack of centralized control:- The component of any ad-hoc networks may join or departure the network dynamically and the network is provision of centralized control on the nodes by leads to rise algorithm's overhead and complexity, like QoS state knowledge must be disseminated proficiently.

Limited power supply:- The mobile nodes are commonly unassisted from confined power provision compared to nodes in the wired network. Statute QoS abrade high power suitable to overhead by the mobile nodes which can sever the node's power immediately.

Security:- It may be considered like a QoS attribute. In absentia enough security, unprocessed access and application may violates the QOS discourse. The nature of relay in wireless networks potentially outcome in high security divestment. The physical passable of infusion is instinctively. So we requirement to design security-aware routing algorithm for ad-hoc networks.

Dynamic topology:-Nodes are free move arbitrarily in any direction with separate momentum speed therefore network topology alteration randomly and onto the unpredictable time. MANET nodes or device on runtime connections routing among themselves as they travel around connections their own network.

Hidden Terminal:-In wireless networking, the hidden node problem or hidden terminal problem rises when a node is manifest by a wireless access point(AP),however not by from other nodes communicating to that access point. This steerage to difference in media access control sub layer.

\section{EVALUATION PARAMETER FOR QOS ROUTING PROTOCOL:}

\subsection{Bandwidth}

Different application needs different bandwidth. In video conferencing we need to send millions of bits per second to refresh a coloring board as long as the overall number of bits into an email may not reach even a million. In order to QoS susceptive there drain a short determinate by required bandwidth, which is the payloads receive by the receiver throughout few specific amount of time, so far as the existing bandwidth by source to destination is not absolutely used from a single traffic stream since to the shared medium, yet routing protocol should confirm until obtain the less necessary data rate proficient from the application layer to the receiver.

\subsection{Packet delay ratio}

PDR is the ratio of the number of delivered data packet of the destination. This illustrates the level of delivered data to the destination.

$\sum$ number of packet receive $/ \sum$ number of packet send.

The highest value to packet delivery ratio implement the better performance of the protocol. The dominant delivery ratio is the ratio of total packets received from the receiver and the total number of packet transfer from the sender. In the congestive network the packets is fallen from the medial node since of the class flooding these minimum packets requirement of retransmitted which let down the network execution a maximum packet delivery ratio is likable.

\subsection{Delay}

The delay of a network classifies how long term this receive a bit of data since passage across is the network by one link or endpoint to other. This is typically scale in several or fractions of seconds. Delay is the total period elapsed between a sender links sends the packet and the receiver the packet, it involves the transmission delay by the sender and interjecent nodes, propagation delay and the waiting series on routers, stand for congestive network delay high dramatically. 


\subsection{Jitter}

Jitter is the variation in delay stand for the packet belongs of the same flow. Since four packets departs at the time 0,1,2,3 and arrive at 20,21,22,23 all have the same delay is 20 unit of time. If above four packets arrive at 21,22,21, and 28, they will have different delays $21,22,19$ and 24 . Variation in the packet delay is the jitter. Example- multimedia communication dealing with jitter.

\subsection{Load balancing}

It is a procedure to distribute workload across several optimum tool practical, high throughput, less response time, increase network life time, and ignore overload. By need multiple paths from load balancing, instead to a single path, can extend reliability through redundancy. The load balancing way is usually ripe from devoted software or hardware, like as a multilayer switch or domain name system server. Unbalanced distribution of traffic oftentimes direction to power depletion to heavily load nodes. The network connectivity sustains leading to frequent disconnection by the network vivisection as much number of number is powered lie down. Load balancing may highly life time to mobile nodes, lower traffic congestions, energy consummation to mobile nodes and end to end packet delays. Routing protocol are ondemand based protocol, load balancing procedure is contribute from the route discovery. Load can be classified as:

\subsubsection{Channel load:}

relate the load onto the channel where several nodes scramble to access the shared media.

\subsubsection{Nodal load:}

represents of a node's activity. Individually it send to how engaged a node is in processing calculation, and so on.

\subsubsection{Neighboring load:}

relates the load presented from the communication activities between neighboring nodes.

\section{CLASSIFICATION OF QOS ROUTING PROTOCOL \\ 4.1 Proactive (QOLSR)}

It is proactive routing protocol. The main concept by this idea in OLSR is the usages of MPR (multi point relays). This MPR send for some router that can farther circulation messages during the whole overflow procedures. OLSR has three main function ,packet onward transmission, neighbor sensing, and topology discovery. Packet onward transmission and neighbor sensing mechanism confer routers with knowledge about the neighbors and after and improve way of flood message in the OLSR network using multi point relay. If less data rates then the high probability unto include the multi point relays. In OLSR have the four message, Hello message, topology control, multiple interface declaration and host and network association. It is a cross layer design outlook which receive in consideration, weighted valency index of the network layer parameter and bit error rate of physical layer parameter, bit error rate is the average error rate of infusion nodes and heavy valency index is the function of nodes unit and nodes capacity(bandwidth). Aim of this protocol is to take path by less bit error rate and maximum weighted connectivity list, path with less bit error rate assures in order that packet retransmission will less and maximum link bandwidth and less series of links are elected.

\subsection{Reactive (AQOR)}

It is protection-oriented procedure to confer QOS warranty. It confer a strategy in order to dynamically framing paths between mobile nodes so that make a Manet. This protocol apply in several methods to permit QoS routing, neighbor discovery and preserve, route exploring route registering (for explored routes). A bandwidth protection network rooted upon the coming to the first packet of a flow, releasing by registered realization(but not reserved) trap free routing mechanism. It elaborate path which confirmed delay and bandwidth compellable, AQOR flood the route request packet. These packet restrain the admissible delay and necessary bandwidth compellable. When an interlude nodes receives the route request(RREQ) packets this will relay only since this assured bandwidth compellable themselves. This protocol usage limited flooding to discover the correct route optimize in term of smallest end to end delay by bandwidth guarantee. A route request packet involves the bandwidth and end to end delay impaction. Let Tmax represent the delay impaction. If a node may verified all the impactions, this will rebroadcast the request of the forward hop and switch to elaborate capacity in order to a less period of 2 Tmax. If several request reach on to the destination, this will forward back a reply packet along every to these routes. Moderate nodes will only send the reply, since they are still into the elaborate state. In-spite of the bandwidth reservation in order to every flow is only activated from the coming to the first data packet from by source node. Delay is measured at the time of route discovery and the route by the less delay is taken from the source.

\subsection{Hierarchical (CEDAR)}

The Core-Extraction Distributed Ad-hoc Routing (CEDAR) algorithm is present in order to QoS routing into ad -hoc networks. Bandwidth knowledge is shown from selected subset nodes along with their link state updates, it identify and ignore congestive caliber of the network. When a link failure, CEDAR's route re-evaluate confirms themselves to the instant neighborhood of the tearing. Core extraction : A set of nodes is selected since make the core in order to balance the topical topology to the nodes into this domain, and also it to functioning the route calculation. The core nodes are selected from approximating a less govern set 1 to the ad-hoc network. Link state Propagation : QoS routing in CEDAR is cognizable from propagating the bandwidth availability knowledge of stationary links to all core nodes. The key idea is in order to the knowledge about stationary maximum bandwidth links can be built-up knowing to nodes far-out away in to the network, as long as knowledge about the mobile and less bandwidth links rest by the local area. Route computation: Route computation firstly maintains a core path by the domain of the source to the domain of destination. By using the directional knowledge prepared offer to search a fractional route by the source to the domain to the furthermost possible node into the core path verifying the requested bandwidth. The node certainly take place the source of the forward iteration. In this the core gives an easiest lessoverflow infrastructure to functioning routing, since the state propagation network ensure availability to link state information into the core nodes in absentia increasing maximum overheads. Route computation should not include the management of state or rather than specific amount to volatile non local state.

\subsection{Predictive Location- (PLBQR)}

This is a location aware QOS routing protocol in that location delay prediction scheme, which is rooted on a location- 
resources information pertaining of the node sending the update. It is a resource knowledge stand for all nodes into the network and their location forecasting rule of procedure are side by side used into the QoS routing conclusion. They have dynamic variations into the topology and resources availability by the maximum degree of mobility to nodes in to the ad-hoc network. By these variations, the topological and routing knowledge used from the present network protocol is adduce outdated very rapidly. It forecast a next physical location rooted on previous location up to date, that is in term to forecast future routes. Update protocol: Every node broadcast that geographical update and resource knowledge periodically and in that content of especially correction. The benefit of these system is the prediction of new location rooted on past location is built-up when there is changes in that geographical location. QoS routing based on to the resource availability at the interlude nodes into the source to destination route is functioning in which is in short supply in the another location rooted routing scheme. Yet correct predication onto the momentum and direction is not built-up when they are dynamic modification in the direction. The transmission is built-up only into a linear way( i.e. angular momentum is kept like as zero). Disadvantage: No resource reservation, In accuracy in delay forecasting .

\subsection{QoS -AODV (QoS enabled AODV)}

It is developed by shayesteh et al is a changes to the traditional AODV routing protocol. QAODV protocol use a weighted parameter is the composite of the several QoS metrics. Therefore here the quality of the node and link by the path are also being carried like as a function in summation to the less number of hop count from source to destination. In order to purpose the route request method of AODV is improved. Since to correcting this impaction, route discovery method present advance overhead of the network, yet the throughput is rising significantly at the charge of these overhead. A bandwidth reservation scheme is unified into the traditional AODV protocol to present QoS-AODV. QoSAODV, heterogeneous another route discovery protocol that avoid the impact to the data link layer, incorporates leak scheduling knowledge to assure end to end bandwidth reservation into a TDMA network. QAODV take different other parameter like as the velocity of node, battery power, the radio susceptibility in the receiver, the antenna benefit, transmission range and bandwidth into consideration. It is rooted on pertinence among delay and bandwidth by transferring the bandwidth need to delay, rooted on the connection among them. There are two matrices, which is combine delay and delay upper bound, are summation onto every routing entrance to solution of the problem that interlude nodes in QAODV are not competent of sending an RREP packet. The packet forward ratio and delay of QAODV are thrice and halved serially from using IQAODV. Presently dynamic bandwidth management (dBM) idea for maximum mobility environments. In this $\mathrm{dBM}$, nodes relay bandwidth reservation needs yet that knowledge is only few of its two hop neighbors. Delay is reduced in dBM.

\subsection{TBP: -(Ticket based Probing)}

Distributed ticket based routing defined by the Chen and Nahrstedt. The main theme of this protocol is to utilize tickets to boundary the number of paths find during route discovery. A ticket is the permit to find a one path. The tickets are used to search the delay unaided or bandwidth unaided routes. In these, when a practicable route is to be maintained among the source and destination rather then source node send a moderate number of probes(routing messages) to some neighboring nodes. This protocol find in order to a least cost delay obligate path or least cost bandwidth obligate path. Its proactive character built-up it unscaleable. This protocol is again improved and a location - aided ticket based protocol (LTBR) is shown which is an unified to location based and ticket based routing, where about the ticket are dynamically generated and directed from location and QoS metric. It given the density of network, and execution of flooding with less routing overhead.

Table.1 Summary of QOS Routing Protocol

\begin{tabular}{|c|c|c|c|c|}
\hline & $\begin{array}{l}\text { Network } \\
\text { Structure }\end{array}$ & $\begin{array}{l}\text { Route } \\
\text { Discovery }\end{array}$ & $\begin{array}{l}\text { Resourc } \\
\text { e } \\
\text { Reservat } \\
\text { ion }\end{array}$ & $\begin{array}{l}\text { QOS } \\
\text { Metrics }\end{array}$ \\
\hline QOLSR & $\begin{array}{l}\text { Hierarchic } \\
\text { al }\end{array}$ & Proactive & Yes & $\begin{array}{l}\text { Throughp } \\
\text { ut, Delay }\end{array}$ \\
\hline AQOR & Flat & Reactive & Yes & $\begin{array}{l}\text { Bandwidt } \\
\text { h, Delay }\end{array}$ \\
\hline CEDAR & $\begin{array}{l}\text { Hierarchic } \\
\text { al }\end{array}$ & $\begin{array}{l}\text { Proactive/React } \\
\text { ive }\end{array}$ & Yes & $\begin{array}{l}\text { Bandwidt } \\
\mathrm{h}\end{array}$ \\
\hline $\begin{array}{l}\text { QOS } \\
\text { Enable } \\
\text { AODV }\end{array}$ & Flat & Reactive & No & $\begin{array}{l}\text { Bandwidt } \\
\text { h, Delay }\end{array}$ \\
\hline TBP & Flat & Reactive & Yes & $\begin{array}{l}\text { Bandwidt } \\
\text { h, Delay }\end{array}$ \\
\hline PLBQR & $\begin{array}{l}\text { Location } \\
\text { Prediction }\end{array}$ & $\begin{array}{l}\text { Proactive/React } \\
\text { ive }\end{array}$ & No & $\begin{array}{l}\text { Bandwidt } \\
\text { h, Delay }\end{array}$ \\
\hline
\end{tabular}

\section{CONCLUSION}

In this paper survey of multiple routing protocol has been finished. These protocol exercise several techniques to verified QoS constraints. Firstly here brief description of protocol has been shown and then there juxtaposition rooted on several metrics like as routing overhead, QoS metrics, load balancing and etc has been given. It presents that so far there many challenges required to clear up to given QoS necessity to the users. This involves be corrected security and reducing power circulation in QoS routing protocol, In absentia offensive routing performance and showing advance to the network. It maintained a route to the destination verified the QoS constraints delay, and bandwidth. These protocol are used to improve the QoS satisfying path. In this focus onto the mainly concept in QoS routing in MANETs and multiple issues that are requirement to be faced during the statute of quality of service. we have built-up the protocols and operation and advantages, drawbacks of this protocol.

\section{REFERENCES}

[1] S.Corson and J.Macker, Mobile Ad hoc Networking (MANET): Routing Protocol Performance Issues and Evaluation Considerations, IETF 2501

[2] S. Singh, M. Woo and C. S. Raghavendra, Power-Aware Routing in Mobile Ad-Hoc Networks,in Proceedings of ACM/IEEE MOBICOM'1998, 1998.

[3] C.R.Lin and J.S. Liu., "QoS routing in ad hoc wireless networks", IEEE J.Select.Areas Commun.,vol.17, pp.1488-1505, 1999. 
[4] C.Perkins, "Ad-hoc On-Demand Distance Vector (AODV) routing", RFC3561[S], 2003.

[5] D.B.Johnson, D.A.Maltz, Y.C.Hu, "The Dynamic Source Routing protocol for mobile ad hoc networks", Internet Draft, 2004.

[6] J.Hong, "Efficient on-demand routing for mobile ad hoc wireless access networks", IEEE journal on selected Areas in Communications 22(2004), 11-35.

[7] Luo Junhai, Xue Lie and Ye Danxia "Research on multicast routing protocols for mobile ad hoc networks" Computer Networks52(2008), 988-997.

[8] S. Chen, Routing Support for Providing Guaranteed Endto-End Quality-of- Service, Ph.D Thesis, University of IL at Urbana-Champaign, 1999.

[9] S. Chakrabarti and A. Mishra, "QoS issues in ad-hoc wireless networks" IEEE Commun. Mag., vol.39,pp.142148, Feb. 2001.

[10] J.N. Al-Karaki and A.E.Kamal, "Quality of Service routing in mobile ad hoc networks: Current and future trends" in Mobile Computing Handbook, CRC publisher,2004.

[11] T.B.Reddy I.Karthigeyan, B.Manoj and C.S.R.Murthy, "Quality of service provisioning in ad hoc wireless networks: a survey of issures and solutions." Vol.4, pp.83-124, 2006.

[12] S.Saunders, "Antennas and Propagation for Wireless Communication System Concept and design",New York, USA: John Wiley and Sons, 1999.

[13] Y.Luo, J.Wang and J.Chen et al., "Algorithm based on mobility prediction and probability for energy efficient multicasting in ad-hoc networks" Computer research and development vol.43(2), pp.231-237,2006.

[14] M. Sipser, Introduction to the Theory of Computation, PWS Publishing, 1997.

[15] Yih Huang, Quality of Service (QoS) Routing A Quick Review of NP-Completeness, technical report,http://www.cs.gmu.edu/ huangyih/756/routing.pdf

[16] Z. Wang and J. Crowcroft, Quality-of-service routing for supporting multimedia applications,

[17] E. Neely and Modiano, Capacity and delay tradeoffs for ad hoc mobile networks, in Proceedings of BroadNets 2004, 2004, pp.428-438.
[18] S.Chakrabarti, and A. Mishra, "QoS issues in ad-hoc wireless networks," IEEE Communication Magazine, Vol. 39, No. 2, pp.142- 148, Feb 2001.

[19] Y.Yang and R.Kravets "Contention-aware admission control for ad hoc networks", IEEE Trans.Mobile Comput., vol.4, 363-377, 2005.

[20] C.R.Lin and J.S. Liu., "QoS routing in ad hoc wireless networks", IEEE J.Select.Areas Commun.,vol.17, pp.1426-11438, 1999.

[21] S.Chen and K.Nahrstedt, "Distributed quality-of-service routin in ad hoc networks" IEEE J.Select.Areas Commun., vol.17, pp.1488-1505, 1999.

[22] A.R.Bashandy, E.K.P.Chong and A.Ghafoor, "Generalized quality-of-service routing with resource allocation" IEEE J.Select.Areas Commun., vol.23, pp.450- 463, 2005.

[23] A.Abradou and W.Zhuang, "A position based QoS routing scheme for UWB mobile ad hoc networks" IEEE J.Select.Areas Commun., vol.24, pp.850-856,2006.

[24] L.Li, C.Li, "A hierarchical QoS multicast routing protocol for mobile ad-hoc networks", Chinese Journal of Electronics 15(4) (2006), 573- 577.

[25] M.S.Kumar, C.Venkatesh, A.M.Ntarajan, "Performance comparison of multicast protocol for physically hierarchical ad-hoc networks using neural Concepts", $7^{\text {th }}$ International conference on Signal Processing Proceedings ICSP, 2004, 1581- 1584.

[26] R.Sivakumar, P.Sinha and V.Bharghavan, "CEDAR: a core-extraction distributed ad hoc routing algorithm" IEEE J.Select. Areas Commun., vol.17, pp.1454-1465, 1999.

[27] L.A.Latif, A.Alliand, C.C. Ooi, "Location based geocasting and forwarding (LGF) routing protocol in mobile-adhoc network", Proceedings on the Advanced Industrial Conference on Telecommunications/Service assurance with partial Intermittent Resources (2005) 536541.

[28] R. Asokan, “A review of Quality of Service (QoS) routing protocols for mobile ad hoc networks," in Proc. IEEE International Conference on Wireless Communication and Sensor Computing(ICWCSC2010),Chennai, India, pp.1- 6 , January 2-4, 2010. 